\title{
WAR DIE ÄLTERE ARRIA PERSIUS’ TANTE? INHALTLICHE UND TEXTKRITISCHE ÜBERLEGUNGEN ZUR VITA PERSI
}

\begin{abstract}
Summary: From the ancient Life of Persius we learn that the poet was a cognatus of Arria the Younger, the wife of Thrasea Paetus. Furthermore, we are told that he was pietatis erga matrem et sororem et amitam exemplo sufficientis. As evidence for his piety towards his mother and his sister the biographer mentions that Persius bequeathed his property to them. But we can also find another act of piety towards a relative mentioned in the poet's biography: a laudatory poem on Arria the Elder. Some considerations about the dates of birth and death of both Arria the Younger and the Elder, Thrasea, and Persius' father corroborate the assumption that Arria the Elder may have been Persius' aunt.
\end{abstract}

Key words: Persius Flaccus, Arria the Elder, Arria the Younger, Thrasea Paetus, Vita Aulis Persi Flacci de commentario Probi Valeri sublata, textual criticism

Als Thrasea Paetus, einer der Oppositionsführer unter Nero, ${ }^{1}$ im Jahr 66 n. Chr. sein Todesurteil annimmt und es im Kreis seiner Verwandten und Freunde selbst an sich vollstreckt, da will seine Frau, die jüngere Arria, dem Beispiel ihrer Mutter folgen und mit ihrem Mann in den Tod gehen; Thrasea aber hält sie davon ab mit der Bitte, der gemeinsamen Tochter Fannia nicht die so wichtige Stütze zu nehmen (vgl. Tac. Ann. 16. 33-35). Eben jene Fannia ist es, die etwa 30 Jahre später die in der Familie tradierten Erinnerungen an ihre Großmutter, die ältere Arria, an Plinius weitergibt (vgl. Plin. Epist. 3. 16). Jene hatte im Jahr 42, als ihr Mann Caecina Paetus, ein Aufständischer unter Claudius, zum Tode verurteilt worden war, sich zuerst den Dolch ins Herz gerammt und diesen mit den unsterblichen Worten 'Paete, non dolet'

${ }^{1}$ Vgl. KunNert, H.: P. Clodius Thrasea Paetus. In RE IV 1 (1900) 99-103; GRIFFIN, M. T.: Nero, The End of a Dynasty (London 1984). Second paperback edition. London 2000, 165f., 171-173. 
(„Paetus, es tut nicht weh!“) an ihn weitergereicht. Diese Geschichte war noch Jahrzehnte später wohlbekannt im römischen Reich (vgl. Plin. Epist. 3. 16. 6; 3. 16. 13; Mart. 1. 13; Cass. Dio 60. 16. 5-7), doch konnte Fannia noch viel mehr über sie erzählen, das erst durch Plinius an die Öffentlichkeit gelangte. ${ }^{2}$ Thrasea, Fannias Vater, war schon damals anwesend, als die ältere Arria sich den Todesstoß zu geben beschlossen hatte; als er sie mit rhetorischem (und geradezu hellseherischem) Geschick fragte: „Willst du denn, dass deine Tochter, wenn ich einmal zum Tode verurteilt werden sollte, mit mir stirbt?“, da antwortete jene: „Wenn sie so lange in ähnlicher Eintracht mit dir gelebt hat wie ich mit meinem Paetus, dann möchte ich es. “3

II.

Thrasea war also bereits im Jahr 42 mit der jüngeren Arria verheiratet. Diese war, so erfahren wir aus der zwar unter Probus' Namen überlieferten, aber wohl auf Sueton zurückgehenden Biographie des Persius, ${ }^{4}$ eine Verwandte des angehenden Satirikers (Vita Pers. 5): Summe dilectus a Paeto Thrasea est, ita ut peregrinaretur quoque cum eo aliquando, cognatam eius Arriam uxorem habente. ${ }^{5}$ Die Reise, die Thrasea

${ }^{2}$ Vgl. Plin. Epist. 3. 16. 2: Multa referebat aviae suae non minora hoc sed obscuriora; Plin. Epist. 3. 16. 13: Videturne haec tibi maiora illo 'Paete, non dolet', ad quod per haec perventum est? Cum interim illud quidem ingens fama, haec nulla circumfert (Text nach MYNORS, R. A. B.: C. Plini Caecili Secundi Epistularum libri decem. Recognovit brevique adnotatione critica instruxit R. A. B. M. Oxford 1963).

${ }^{3}$ Plin. Epist. 3. 16. 10: Quin etiam, cum Thrasea gener eius deprecaretur, ne mori pergeret, interque alia dixisset: 'Vis ergo filiam tuam, si mihi pereundum fuerit, mori mecum?', respondit: 'Si tam diu tantaque concordia vixerit tecum quam ego cum Paeto, volo.'

${ }^{4}$ Zur wahrscheinlich suetonischen Autorschaft der Vita Aulis Persi Flacci de commentario Probi Valeri sublata vgl. GLÄSER, F.: Quaestiones Suetonianae de vitis Persii Lucani Horatii. Diss. Breslau 1911, 3-28; SALLMANN, K.: §404. C. Suetonius Tranquillus, B.4. Literarhistorische Schriften. In SALLMANN, K. (Hg.): Handbuch der lateinischen Literatur der Antike 4. Die Literatur des Umbruchs von der römischen zur christlichen Literatur: 117 bis 284 n. Chr. München 1997, 27-40, 37; SCHMIDT, P. L.: Aulus Persius Flaccus. In DNP 9 (2000) 618-620, 618; PARKER, H. N. - BRAUND, S.: Imperial Satire and the Scholars. In BRAund, S. - Osgood, J. (Hgg.): A Companion to Persius and Juvenal. Chichester 2012, 436-464, 438f. Für den aus Beirut stammenden Grammatiker Valerius Probus als Autor argumentiert Aistermann, J.: De M. Valerii Probi Berytii vita et scriptis. Diss. Bonn 1909, 45-57. L. TAKÁCS (The ancient biography of Aules Persius Flaccus or the so-called Vita Persii de commentario Probi Valeri sublata. ACD 43 [2007] 183-187, 186f.) dagegen meint, die Vita stamme weder von Probus noch von Sueton, sondern von einem Freund des Dichters aus dem Umkreis des Quintilian, der ebenso wie Persius mit dem Historiker Servilius Nonianus verkehrte (vgl. Quint. Inst. 10. 1. 102; Vita Pers. 5); doch dazu s. u. Anm. 26. Die Kapitelzählung folgt der Nummerierung von F. LEO (A. Persii Flacci, D. Iunii Iuvenalis, Sulpiciae Saturae. Recognovit O. Jahn, post F. Buecheleri iteratas curas editionem quartam curavit F. L. Berlin 1910, 64-67); der Text wird an den umstrittenen Stellen, an denen sich die aktuellen Ausgaben von W. V. Clausen (A. Persi Flacci et D. Iuni Iuvenalis Saturae. Edidit brevique adnotatione critica denuo instruxit W. V. C. Oxford 1992) und W. KISSEL (A. Persius Flaccus, Saturarum liber. Berlin 2007) sehr zurückhaltend im Umgang mit alten wie neuen Konjekturen zeigen, stets diskutiert.

${ }^{5}$ Dazu, dass das gewöhnlich als Einleitung dieses Satzes gedruckte decem fere annis nicht zu diesem Satz gehört, sondern als Ablativus comparationis im vorangehenden Satz erklärt, um wie viele Jahre Persius jünger war als seine darin genannten Freunde und Mitschüler unter Cornutus, vgl. PARATORE, E.: Persio e Lucano. RCCM 5 (1963) 88-130, 102-105 sowie TAKÁCS, L.: $A$ Vita Persii de commentario Probi Valeri sublata. Elemzés és kommentár [Analyse und Kommentar]. Piliscsaba 2012, $115 f$. 
und Persius gemeinsam unternommen haben, fand möglicherweise ihren Niederschlag in einem (nicht erhaltenen) Hodoeporicon, mit dem der junge Dichter das Iter Siculum des Lucilius (Buch III) und Horazens Iter Brundisinum (Sat. 1. 5) nachgeahmt haben mag. ${ }^{6}$

Ein anderes Gedicht, das Persius in seiner Jugend schrieb, ist ein Loblied auf die ältere Arria, die Schwiegermutter seines Freundes Thrasea (Vita Pers. 8): Scripserat in pueritia ... et paucos in socrum Thraseae [in Arriam matrem] versus, quae se ante virum occiderat.

Diese Stücke sind allerdings vom literarischen Nachlassverwalter des Persius, seinem Lehrer Annaeus Cornutus, vernichtet worden. ${ }^{8}$ Ferner erfahren wir über den Nachlass des Persius, dass dieser sein Vermögen von ca. 2000000 Sesterz seiner Mutter und seiner Schwester hinterlassen hat (Vita Pers. 7): Reliquit cira HS vicies matri et sorori.

Persius' Beziehung zu den Frauen seiner Familie war von enger Liebe und tiefem Familiensinn geprägt; der Biograph meint gar, man könne sich ihn darin zum Vorbild nehmen (Vita Pers. 6): Fuit ... pietatis erga matrem et sororem et amitam exemplo sufficientis.

Für die pietas erga matrem et sororem wird vom Biographen mit dem Erbe ein Beispiel geliefert. Wenn er nun aber zu allen drei genannten Frauen eine vorbildliche Beziehung hegte, warum bedachte er dann nur zwei von ihnen mit dem Erbe? Die Antwort liegt auf der Hand: Die amita, d. h. Tante väterlicherseits, des Persius wird wohl bereits vor ihm gestorben sein. Und es wird in der Vita schließlich noch ein anderer Akt der pietas gegenüber einer Verwandten erwähnt: das Loblied auf die ältere Arria - wenn die jüngere Arria eine cognata des Dichters war, so gehörte ihre

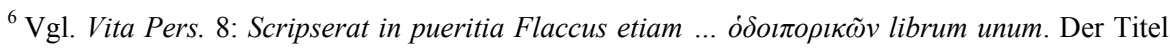
ist von P. Pithou (A. Persii Satyrarum liber I, D. Iunii Iuvenalis Satyrarum lib. V, Sulpiciae Satyra I. Cum veteribus commentariis nunc primum editis. Ex bibliotheca P. Pithoei IC., cuius etiam notae quaedam adiectae sunt. Paris 1585) für verderbtes opericon hergestellt; er wird aus den genannten Gründen, dem Nacheifern der satirischen Vorbilder und der Information über eine vom Biographen als erwähnenswert erachtete Reise, von der Forschung nahezu einhellig akzeptiert. Setzt man diese Reise in die Jugendzeit des Dichters (vgl. scripserat in pueritia), so wird die Annahme, die Freundschaft zwischen den beiden sei in die letzten Lebensjahre des Dichters zu datieren (vgl. decem fere annis summe dilectus a Paeto Thrasea est; so etwa KUNNERT (Anm. 1) 101: „Der jungen Generation, die hier, erfüllt von stoisch-republicanischen Ideen, heranwuchs, gehörte auch Persius an, der mit Thrasea an die zehn Jahre (52-62) verkehrte und ihn auf einer Reise begleitete, welche er nach des Lucilius und Horatius Vorbild dichterisch behandelte"), als haltlos erwiesen. Die Tatsache, dass Thrasea bereits 42 mit einer cognata des Persius verheiratet war, tut ihr Übriges, um den Bezug von decem fere annis auf summe dilectus est unwahrscheinlich zu machen (s. o. Anm. 5).

${ }^{7}$ Überliefert ist verderbtes paucos sororum Thrasiae in Arriam matrem versus; die Emendation von sororum Thrasiae zu in socrum Thraseae stammt von I. CASAUBON (Auli Persi Flacci Satirarum liber. I. C. recensuit et commentario libro illustravit. Paris 1605); bei der Athetese von in Arriam matrem folge ich O. JAHN (Auli Persii Flacci Satirarum liber. Cum scholiis antiquis edidit O. I. Leipzig 1843, 237 Fn. 7): Es ist wahrscheinlicher, dass die Umschreibung socrus Thraseae mit der konkreten Angabe Arria mater kommentiert wurde, als dass zu einer konkreten Benennung der Person eine abstraktere Umschreibung hinzugegeben worden sein soll.

${ }^{8}$ Vgl. Vita Pers. 8: Omnia ea, auctor fuit Cornutus matri eius, ut aboleret. 
Mutter freilich auch zur Familie. War also die ältere Arria möglicherweise die Tante väterlicherseits, amita, des Persius?

III.

Einige Überlegungen zu den Lebensdaten der genannten Personen mögen diese These erhärten.

Von der jüngeren Arria wissen wir, dass sie nach dem Tod Domitians (96 n. Chr.) noch am Leben war (vgl. Plin. Epist. 9. 13. 5), kurz darauf aber wohl gestorben ist (vgl. Plin. Epist. 7. 19. 9). Da sie aber im Jahr 42 beim Tod ihrer Mutter bereits mit Thrasea verheiratet war (vgl. Plin. Epist. 3. 16. 10) und beim Tod ihres Mannes im Jahr 66 eine ihrerseits verheiratete Tochter hatte (vgl. Tac. Ann. 16. 35. 1), kann man bei einem üblichen Heiratsalter von etwa 20 Jahren $^{9}$ für sie eine realistische Lebensdauer von etwa 22 bis $97 \mathrm{n}$. Chr. annehmen.

Da ihre Tochter Fannia wohl schon vor der Bekleidung des Volkstribunats ihres Mannes im Jahr 56 verheiratet war, ${ }^{10}$ ließe sich das Geburtsjahr der jüngeren Arria wohl noch um einige Jahre früher ansetzen. Fannia scheint dagegen schon sehr jung verheiratet gewesen zu sein: Thrasea macht sich schließlich Sorgen, der Tod der Mutter würde sie einer ungemein wichtigen Stütze (subsidium unicum) berauben (vgl. Tac. Ann. 16. 34. 2) - die ältere Arria dagegen hat ihre Tochter im Jahr 42 bereits schon auf eigenen Beinen im Leben stehen sehen; bei Thraseas Versuch, die ältere Arria vom Selbstmord abzuhalten, nennt dieser zwar ihre Tochter, erwähnt aber noch keine Enkelin; Fannia scheint also erst nach 42 geboren zu sein - das gesetzliche Mindestalter einer Frau zum Zeitpunkt ihrer ersten Eheschließung betrug 12 Jahre; ${ }^{11}$ folglich ist es nicht ausgeschlossen, Fannia als etwa 43 geboren anzunehmen. Für die jüngere Arria ließe sich dann eine Lebenszeit von etwa 22-97 halten.

Thrasea lässt vor dem Prozess im Jahr 66, der schließlich zu seinem Todesurteil führen wird, verlauten, er „habe sein Leben gelebt“ (sibi actam aetatem, Tac. Ann. 16. 25. 5); da er zudem im Jahr 56 Suffektkonsul war, ${ }^{12}$ dürfen wir sein Geburtsjahr - unter der Annahme, dass zu seiner Zeit immer noch ein Richtwert von 43 Jahren als Mindestalter zur Bekleidung des Konsulats eingehalten worden ist ${ }^{13}-$ auf etwa

\footnotetext{
${ }^{9}$ Vgl. TREgGiari, S.: Ehe, III. Rom (Übers.: T. RAUBITSCHEK). In DNP 3 (1997) 896-899, 897: „Zum Zeitpunkt ihrer ersten Eheschließung, die durch Gesetz für Mädchen ab einem Alter von 12 Jahren, für junge Männer ab 14 Jahren erlaubt war, waren Frauen normalerweise knapp 20, Männer knapp 30 Jahre alt. In der polit. Führungsschicht war das Heiratsalter oft niedriger, besonders seit dem frühen Prinzipat, als das Mindestalter für die Bekleidung polit. Ämter herabgesetzt wurde und die Ehegesetze es Kandidaten darüber hinaus erlaubten, für jedes Kind das erforderliche Mindestalter um ein Jahr zu reduzieren. “

${ }^{10}$ Vgl. Kappelmacher, A.: Fannia. In RE VI 2 (1909) 1995; GAHEIS, A.: C. Helvidius Priscus. In RE VIII 1 (1912) 216-221, 218-220.

${ }^{11}$ Vgl. TREgGiari (Anm. 9) 897 (s. o.).

${ }^{12}$ Vgl. KunNerT (Anm. 1) 100.

${ }^{13}$ Vgl. Cic. de off. 2. 39; Brut. 323; de leg. agr. 2. 3; dazu KÜBLER, B.: Magistratus. In RE XIV 1 (1928) 400-437, 406; zur schrittweisen Auflösung der Altersgrenzen in der Kaiserzeit vgl. allerdings ebd. 431 sowie TREGGiARI (Anm. 9) 897 (s. o.).
} 
13 n. Chr. oder früher schätzen. Er wird also etwas älter als seine Frau gewesen sein; dies passt auch mit dem für Männer üblichen Heiratsalter von etwa 30 Jahren zusammen. ${ }^{14}$ Wir können also folgern, dass die Freundschaft zwischen ihm und Persius eine generationsübergreifende gewesen ist, der im Jahr 34 geborene Dichter (vgl. Vita Pers. 1) sich gewissermaßen ,des väterlichen Wohlwollens Thraseas erfreute“15 - sie kannten sich schließlich mindestens seit 42, als Thrasea seine mit Persius verwandte Schwiegermutter vom Selbstmord abhalten wollte (s. o.).

Seinen echten Vater verlor Persius bereits als kleines Kind (pupillus, vgl. Vita Pers. 3), seinen Stiefvater kurz darauf (vgl. Vita Pers. 3: Fulvia Sisennia nupsit postea Fusio equiti Romano et eum quoque extulit intra paucos annos).

Nun ist aber der Satz, der vom Tod des Vaters berichtet, in der Form, in der er überliefert ist, wohl mit Sicherheit als verderbt zu betrachten (Vita Pers. 3): Pater eum (bzw. eius) Flaccus pupillum reliquit moriens annorum fere sex.

Eigenartig an diesem Satz ist nicht nur, dass die auf den Vater bezogenen Wörter pater ... Flaccus ... reliquit moriens stets von solchen, die den Jungen bezeichnen, eum ... pupillum, unterbrochen sind, sondern auch die Doppelung von reliquit und moriens, obwohl doch relinquere allein schon ,sterbend hinterlassen“ heißt (vgl. etwa Vita Pers. 7; Suet. Vita Ter. 6; Suet. Gramm. 11. 1: pupillum relictum); schließlich erwartet man nach moriens das Alter des Sterbenden, nicht das des hinterlassenen Kindes. ${ }^{16}$

Die erste Hälfte des Satzes hat man zu bereinigen versucht, indem man wahlweise eum (Bücheler 1886) oder Flaccus tilgte (Bücheler 1893). ${ }^{17}$ Nahezu ungehört verhallt ist der sehr elegante Emendationsversuch von O. Jahn (1851), der das im Codex Parisinus 8272 überlieferte eius auf ein ursprüngliches Gaius zurückführt. ${ }^{18}$

Die zweite Hälfte dagegen hat man sich, sofern man nicht moriens annorum fere sex komplett athetiert hat (Korzeniewski 1965), ${ }^{19}$ so, wie sie ist, hinzunehmen genötigt gefühlt. Dabei spricht noch etwas dagegen, dass die so mitgeteilte Nachricht, Persius habe seinen Vater mit sechs Jahren verloren, richtig sein kann. In einer Erinnerung an seine Schulzeit erzählt Persius, er habe sich oftmals, als er öffentlich eine Deklamationsübung vortragen sollte, die Augen mit Öl beträuft, um sich vor dem Auftritt zu drücken (Pers. 3. 44-47): Saepe oculos, memini, tangebam parvus olivo, / grandia si nollem morituri verba Catonis / dicere non sano multum laudanda magistro, / quae pater adductis sudans audiret amicis. Obwohl solche Auftritte mit Übungsreden zwar erst in der Rhetorikschule (die man erst mit etwa 16 Jahren besuchte) üblich waren, sind sie für die Grammatikschule dennoch nicht undenkbar,

${ }^{14}$ Vgl. TRegGiari (Anm. 9) 897 (s. o.).

${ }^{15}$ So fasst E. KoestermanN (Cornelius Tacitus, Annalen, Band IV: Buch 14-16. Erläutert und mit einer Einleitung versehen von E. K. Heidelberg 1968, 408) die Beziehung treffend zusammen.

${ }^{16}$ Vgl. KorZenIEwSKI, D.: Rez. E. Paratore, La poetica di Persio, Rom 1964. Gnomon 37 (1965) 774-777, 775.

${ }^{17}$ Vgl. BÜChELER, F.: A. Persii Flacci, D. Iunii Iuvenalis, Sulpiciae Saturae. Recognovit O. Jahn, editio altera curam agente F. B. Berlin 1886, 54: pater Flaccum pupillum reliquit; bzw. BÜCHELER, F.: ... editio tertia. Berlin 1893, 58: pater eum [Flaccus] pupillum reliquit.

${ }^{18}$ Vgl. JAHN, O.: A. Persii Flacci Satirarum liber. Recensuit O. I. Leipzig 1851, 37 im krit. App.

${ }^{19}$ Vgl. KORZENIEWSKI (Anm. 16) 775. 
Persius bezeichnet sich schließlich als parvus. ${ }^{20}$ Der bei der genannten Deklamation anwesende pater muss also, als Persius mit etwa 12 Jahren Unterricht bei Remmius Palaemon erhielt (vgl. Vita Pers. 4), zu dieser Zeit noch gelebt haben; er kann damit nicht Persius als sechsjähriges Kind zurückgelassen haben. ${ }^{21}$ Doch hat es auch in diesem Fall bereits einen Emendationsvorschlag gegeben, der ebensowenig Beachtung fand wie derjenige von O. Jahn (1851) zur ersten Hälfte des Satzes: L. Herrmann (1955) schlug vor, moriens annorum fere sex $<$ aginta $>$ zu lesen. ${ }^{22}$

Der so zu rekonstruierende Satz Pater C. Flaccus pupillum reliquit moriens annorum fere sexaginta erregt weder hinsichtlich seiner Wortstellung noch bezüglich seines Inhalts Anstoß; zudem ist er näher an der Überlieferung als jegliche Emendation durch Athetese einzelner Wörter oder ganzer Wortgruppen (s. o.).

Für Persius' Vater wäre so also eine Lebenszeit von etwa 15 v. Chr. bis 46 n. Chr. zu erschließen; seinen Sohn Aules hätte er dann etwa in seinen späten 40ern gezeugt das ist nicht auszuschließen.

Die ältere Arria hatte nun, nachdem sie tam diu tantaque concordia mit Caecina Paetus gelebt hatte, im Jahr 42 eine erwachsene und verheiratete Tochter (vgl. Plin. Epist. 3. 16. 10): Lassen wir sie so wie die jüngere Arria auch im üblichen Alter von etwa 20 Jahren den Bund der Ehe eingegangen sein, so kommen wir auf etwa $2 \mathrm{n}$. Chr. als terminus ante quem für ihre Geburt; lassen wir sie ihre Tochter nicht mit 20, sondern erst mit 30 Jahren geboren haben, so können wir ihr Geburtsjahr auch realistisch um 9 v. Chr. ansetzen, womit sie nur knapp jünger als Persius' Vater wäre.

Wollen wir in ihr nun aber die amita des Persius, also die Schwester seines Vaters sehen, so bleibt ein letztes Hindernis zu umschiffen: Persius' Vater hat - gemäß der vorgestellten Konjektur von O. Jahn (1851) - wohl den Namen C. Persius Flaccus getragen, Arria dagegen muss aus der gens Arria stammen. Die beiden können also bestenfalls Halbgeschwister sein: Dann würden sie sich die Mutter teilen, aber von verschiedenen Vätern, einem Persius bzw. einem Arrius, gezeugt worden sein. Doch ist auch dies beileibe nichts Unmögliches.

IV.

Die hier vorgestellte Rekonstruktion der Verwandtschaftsverhältnisse zwischen Persius und Arria, der jüngeren wie der älteren, ist zweifelsohne höchst spekulativ. Doch ist sie hinsichtlich der Lebensdaten aller darin verwickelten Personen durchaus plausibel und erklärt zudem, inwieweit Persius sanguine et affinitate primi ordinis viris coniunctus (Vita Pers. 2) und die jüngere Arria seine cognata (Vita Pers. 5) gewesen

${ }^{20}$ Vgl. KiSSEL, W.: Aules Persius Flaccus, Satiren. Herausgegeben, übersetzt und kommentiert von W. K. Heidelberg 1990, 420, dem ich auch hinsichtlich des Persius-Textes folge.

${ }^{21}$ KISSEL (Anm. 20) 421 versucht, dieses Problem zu lösen, indem er annimmt, „daß Persius auch den zweiten Ehemann seiner Mutter (Vita Z. 8f.) in kindlicher Anhänglichkeit pater nennen und dieser, obwohl ebenfalls bald verstorben, die ersten rhetorischen Versuche seines privignus noch mit gespanntem Interesse verfolgen konnte".

${ }^{22}$ Vgl. HerrmanN, L.: Perse écolier. RBPh 33 (1955) 317-319, 317. 
ist: Sie wäre seine Halbcousine. Darüber hinaus füllt die hier vorgestellte These eine Phantomgestalt in der Vita Persi, die amita, mit Leben: Der junge Persius war ihr sehr verbunden, konnte ihr aber nichts vererben, da sie bereits in seiner Kindheit gestorben war; als Akt seiner pietas ihr gegenüber (vgl. Vita Pers. 6) schrieb er ein Loblied auf sie (vgl. Vita Pers. 8).

Man mag es dem Biographen nachsehen, nur diese wenigen spärlichen Hinweise auf die genauen Verwandtschaftbeziehungen gegeben zu haben - vielleicht befand er schlicht, dass cognata deutlich enger klingt als eine genaue Beschreibung des Sachverhalts, der etwa mit der (nicht belegten) Wendung amitina alio avo zu umschreiben wäre (was aber eher in einem Gesetzestext denn in einer Literatenbiographie seinen angemessenen Platz hätte) ${ }^{23}$

Sollte Sueton tatsächlich der Autor der Vita Persi sein, ${ }^{24}$ so könnte er die Informationen über diese Familie von seinem Freund Plinius (vgl. Plin. Epist. 1. 18; 3. 8; 5. 10; 9. 34) ${ }^{25}$ erhalten haben, der persönlichen Kontakt zu Fannia, der Enkelin der älteren Arria, sowie zur jüngeren Arria selbst pflegte (vgl. Plin. Epist. 3. 11. 3; 3. 16; 7. 19; 9. 13). ${ }^{26}$ Diese beiden könnten auch durchaus noch die nur im engsten Familienkreis bekannten in socrum Thraseae versus (Vita Pers. 8) des Persius gehört haben.

\author{
Markus Stachon \\ Institut für Klassische und Romanische Philologie \\ Rheinische Friedrich-Wilhelms-Universität Bonn \\ Am Hof 1e \\ 53113 Bonn \\ Deutschland
}

${ }^{23}$ Vgl. die Belegstellen bei Vollmer, F.: amitinus, - $a$. In ThLL I (1905) 1920.

${ }^{24}$ S. o. Anm. 4.

${ }^{25}$ Dazu vgl. Winsbury, R.: Pliny the Younger. A Life in Roman Letters. London 2014, 179-182.

${ }^{26}$ Hält man sich vor Augen, dass Plinius sowohl zu Arria selbst als auch zu Sueton engen Kontakt hatte, so entkräftet sich die Forderung von AISTERMANN (Anm. 4) 56 und TAKÁCS (Anm. 4) 186, der Biograph müsse die Vita sehr kurz nach dem Tod des Dichters verfasst haben, da er sonst nicht mehr an solch gute Quellen hätte gelangen können, als Argument für vor-suetonische Autorschaft selbst. 